PROCEEDINGS OF THE

AMERICAN MATHEMATICAL SOCIETY

Volume 131, Number 9, Pages 2809-2817

S 0002-9939(03)06846-

Article electronically published on January 2, 2003

\title{
INTERPOLATING SEQUENCES IN HARMONICALLY WEIGHTED DIRICHLET SPACES
}

\author{
ANTÓNIO SERRA
}

(Communicated by Juha M. Heinonen)

\begin{abstract}
We describe the interpolating sequences and weak interpolating sequences for the multiplier algebras of harmonically weighted Dirichlet spaces $D(\mu)$ when $\mu$ is a finitely atomic measure.
\end{abstract}

\section{INTRODUCTION}

Let $A$ be a Banach algebra of (necessarily bounded) functions defined on the open unit disk $\mathbb{D}$ in the complex plane. A sequence $\left(z_{n}\right)_{n \in \mathbb{N}}$ in $\mathbb{D}$ is called an interpolating sequence (for $A$ ) if the operator $T: A \rightarrow l^{\infty}$ defined by

$$
(T f)_{n}=f\left(z_{n}\right)
$$

is surjective, and it is called a weak interpolating sequence (for $A$ ) if there exists a bounded sequence $\left(f_{n}\right) \subset A$ such that $f_{n}\left(z_{m}\right)=\delta_{m n}$.

We examine here the case where $A$ is the multiplier algebra of a certain kind of weighted Dirichlet space.

Let $\left(z_{n}\right)$ be a Blaschke sequence in $\mathbb{D}$. For each $n$ define

$$
\beta_{n}(z)=\prod_{k \neq n} \frac{\left|z_{k}\right|}{z_{k}} \frac{z_{k}-z}{1-\overline{z_{k}} z} .
$$

L. Carleson proved ([2], see [3] , 4] , [10] and references there) that such a sequence $\left(z_{n}\right)$ is an interpolating sequence for $H^{\infty}$ if and only if

$$
\left|\beta_{n}\left(z_{n}\right)\right| \text { is bounded away from zero. }
$$

This shows that for $H^{\infty}$, interpolating sequences coincide with weak interpolating sequences.

A condition which is necessary for $\left(z_{n}\right)$ to be an interpolating sequence for $H^{\infty}$ is that

$$
\forall_{f \in H^{2}}\left(\left(1-\left|z_{n}\right|^{2}\right)^{\frac{1}{2}} f\left(z_{n}\right)\right) \in l^{2},
$$

and a key step in Carleson's work 2 is to prove that $(\mathbb{C})$ implies $(\mathbb{N})$. A proof using Hilbert space techniques was introduced in [10] and can also be found in [4].

Received by the editors May 3, 2001 and, in revised form, April 3, 2002.

2000 Mathematics Subject Classification. Primary 30E05; Secondary 46E20.

Key words and phrases. Interpolating sequences, weighted Dirichlet spaces.

This work was fully supported by the PRAXIS XXI program, scholarship BD/11064/97, F.C.T., Lisbon, Portugal. 
Let $\mu$ be a positive finite Borel measure on $\partial \mathbb{D}$, the unit circle, let $P(\mu)(z)$ denote its Poisson integral evaluated at $z$, and let $d A$ denote normalized Lebesgue area measure. In [6], Stefan Richter introduced the Hilbert spaces $D(\mu)$, consisting of the functions $f$ holomorphic in $\mathbb{D}$ for which

$$
D_{\mu}(f)=\int\left|f^{\prime}\right|^{2} P(\mu) d A
$$

is finite, and used them to give a complete classification of 2-isometries.

We will first study (weak) interpolating sequences for the case when $\mu$ is the unit mass at 1 and then show how the results generalize to finitely atomic measures.

In [7], it was proved that $D\left(\delta_{1}\right)$ is the space of all holomorphic functions $f$ defined on $\mathbb{D}$ of the form

$$
f(z)=a+(z-1) f_{1}(z)
$$

for some $a \in \mathbb{C}$ and $f_{1} \in H^{2}$, equipped with the inner product

$$
\langle f, g\rangle=\langle f, g\rangle_{H^{2}}+\left\langle f_{1}, g_{1}\right\rangle_{H^{2}}
$$

a different proof was given in 9 .

One can see that in (1.2) $a$ will be the radial limit $f(1)$. The functional that sends $f$ to $f(1)$ is a bounded linear functional on $D\left(\delta_{1}\right)$. Also, given $f \in H^{2}$ and $\zeta \in \partial \mathbb{D}$, define

$$
D_{\zeta}(f):=\left\|\frac{f-f(\zeta)}{z-\zeta}\right\|_{H^{2}}^{2}
$$

where $f(\zeta)$ denotes the radial limit of $f$ at $\zeta$ if it exists (the norm on the right side is interpreted as $\infty$ if it does not exist). Thus $f \in D\left(\delta_{1}\right)$ if and only if $D_{1}(f)$ is finite and in that case

$$
\|f\|_{D\left(\delta_{1}\right)}^{2}=\|f\|_{H^{2}}^{2}+D_{1}(f) .
$$

Let $M\left(\delta_{1}\right)$ denote the multiplier algebra of $D\left(\delta_{1}\right)$, i.e., the space of functions $\phi$ holomorphic on $\mathbb{D}$ such that for every $f \in D\left(\delta_{1}\right)$ one has $\phi f \in D\left(\delta_{1}\right)$. It can be easily seen that the algebra $M\left(\delta_{1}\right)$ consists of those functions in $D\left(\delta_{1}\right)$ that are bounded. Given $\phi \in M\left(\delta_{1}\right)$ let $M_{\phi}$ denote the operator of multiplication by $\phi$ on $D\left(\delta_{1}\right)$. It is a direct consequence of the closed graph theorem that such operators must be bounded. We will also denote by $M\left(\delta_{1}\right)$ the set of these operators. Being the commutant of $M_{z}$ in $\mathcal{B}\left(D\left(\delta_{1}\right)\right), M\left(\delta_{1}\right)$ is a Banach algebra. If $\phi \in M\left(\delta_{1}\right)$, we will denote by $\|\phi\|_{M}$ the norm of the operator $M_{\phi}$ in $\mathcal{B}\left(D\left(\delta_{1}\right)\right)$.

The elements $\phi$ of $M\left(\delta_{1}\right)$ satisfy $\|\phi\|_{\infty} \leq\|\phi\|_{M}$. In fact, being characters of the Banach algebra $M\left(\delta_{1}\right)$, the point evaluations must be functionals of norm one. Another way to prove this is to observe that for each element $w$ of the range of a multiplier $\phi$ of a Hilbert space with reproducing kernel $k$, the conjugate $\bar{w}$ is an eigenvalue of the adjoint operator $M_{\phi}^{*}$ associated with the eigenvector $k(\cdot, w)$.

For $z \in \mathbb{D}$ define

$$
P(z)=\frac{1-|z|^{2}}{|1-z|^{2}}
$$

that is, $P(z)$ is the Poisson integral of the unit mass at 1 evaluated at $z$.

We prove that a sequence $\left(z_{n}\right) \subset \mathbb{D}$ is an interpolating sequence (resp. a weak interpolating sequence) for $M\left(\delta_{1}\right)$ if and only if $\left(z_{n}\right)$ satisfies (C) and the sequence $\left(P\left(z_{n}\right)\right)$ is in $l^{1}\left(\right.$ resp. $\left.l^{\infty}\right)$. 
Further we show that when $\mu$ is a finite sum of point masses at $\lambda_{1}, \ldots, \lambda_{N}$, the interpolating sequences (resp. weak interpolating sequences) are precisely those which belong to the respective category for each of the summands.

\section{INTERPOLATING SEQUENCES}

The main result in this section is Theorem 2.4 below. First, for completeness, we give self-contained proofs of some facts which will be used.

We will need the following lemma.

Lemma 2.1. Let $f \in D\left(\delta_{1}\right)$ and $a \in \mathbb{D}$. Let

$$
b(z)=\frac{a-z}{1-\bar{a} z} .
$$

Then

$$
\|b f\|_{D\left(\delta_{1}\right)}^{2}=\|f\|_{D\left(\delta_{1}\right)}^{2}+|f(1)|^{2} P(a) .
$$

Proof. It is enough to prove that

$$
D_{1}(b f)=D_{1}(f)+D_{1}(b)|f(1)|^{2}
$$

and $D_{1}(b)=P(a)$. We have that

$$
\frac{b(z)-b(1)}{z-1}=\frac{|a|^{2}-1}{1-\bar{a}} \frac{1}{1-\bar{a} z}
$$

and the second factor is the Szegö kernel at $a$, so the second equality follows. As to the first, we have

$$
\frac{b(z) f(z)-f(1) b(1)}{z-1}=b(z) \frac{f(z)-f(1)}{z-1}+f(1) \frac{b(z)-b(1)}{z-1}
$$

and from (2.3) we see that the two summands on the right are orthogonal in $H^{2}$, and the sum of their square norms equals the right-hand side of (2.2).

The identity (2.2) is proved in a more general form in [7].

The following proposition is part of a larger theorem in [8] (Theorem 6, page $89)$.

Proposition 2.2. Given a sequence $\left(z_{n}\right)$ in $\mathbb{D}$, the Blaschke product

$$
B(z)=\prod_{n \geq 1} \frac{\left|z_{n}\right|}{z_{n}} \frac{z_{n}-z}{1-\overline{z_{n}} z}
$$

converges and belongs to $D\left(\delta_{1}\right)$ if and only if $\left(z_{n}\right)$ satisfies

$$
\left(P\left(z_{n}\right)\right) \in l^{1}
$$

and in that case $|B(1)|=1$ and $\|B\|_{D\left(\delta_{1}\right)}^{2}=1+\sum_{n \geq 1} P\left(z_{n}\right)$.

Proof. Assume that $B \in D\left(\delta_{1}\right)$. For $n \geq 1$ define

$$
B_{n}(z)=\prod_{k=1}^{n} \frac{\left|z_{n}\right|}{z_{n}} \frac{z_{n}-z}{1-\overline{z_{n}} z} .
$$

Iterating Lemma 2.1 we have

$$
\|B\|_{D\left(\delta_{1}\right)}^{2}=\left\|\frac{B}{B_{n}}\right\|_{D\left(\delta_{1}\right)}^{2}+\sum_{k=1}^{n} P\left(z_{k}\right)
$$


for all $n \geq 1$ (we have $\left|B(1) / B_{n}(1)\right|=1$ ). So $(\underline{\mathrm{L}})$ holds, proving necessity. To establish sufficiency, assume that (L) holds; then $\left(z_{n}\right)$ must be a Blaschke sequence. Also, $\left(B_{n}\right)$ is a sequence in $D\left(\delta_{1}\right)$, since these functions are holomorphic on the closed disc. Moreover, $\left|B_{n}(1)\right|=1$. As is well known, the sequence $\left(B_{n}\right)$ converges to $B$ in $H^{2}$ norm. In fact,

$$
\begin{gathered}
\left\|B_{n}-B\right\|_{H^{2}}^{2}=\left\|1-\frac{B}{B_{n}}\right\|_{H^{2}}^{2} \\
=2-2 \operatorname{Re}\left\langle 1, \frac{B}{B_{n}}\right\rangle_{H^{2}}=2-2 \frac{B(0)}{B_{n}(0)} \rightarrow 0 .
\end{gathered}
$$

Next we assert that

$$
\left(\frac{B_{n}(z)-B_{n}(1)}{z-1}\right)
$$

is a fundamental sequence in $H^{2}$. We again use Lemma 2.1 to compute

$$
D_{1}\left(B_{n}-B_{m}\right)=D_{1}\left(B_{m}\left(\frac{B_{n}}{B_{m}}-1\right)\right)=D_{1}\left(\frac{B_{n}}{B_{m}}-1\right)+\left(\sum_{k=1}^{m} P\left(z_{k}\right)\right)\left|\frac{B_{n}(1)}{B_{m}(1)}-1\right|^{2} \text {. }
$$

The sum on the right is bounded. Also, $B_{n} / B_{m}$ approaches 1 in $H^{2}$ norm as $m, n \rightarrow \infty$ and

$$
D_{1}\left(\frac{B_{n}}{B_{m}}-1\right)=D_{1}\left(\frac{B_{n}}{B_{m}}\right)=\sum_{k=m+1}^{n} P\left(z_{n}\right),
$$

which converges to zero when $m$ and $n$ go to infinity. This shows that $B_{n} / B_{m} \rightarrow 1$ in $D\left(\delta_{1}\right)$ and since evaluation at 1 is a bounded functional in $D\left(\delta_{1}\right)$, we have that $B_{n}(1) / B_{m}(1)$ converges to 1 when $m, n \rightarrow \infty$. Therefore our assertion is established, and we conclude that $\left(B_{n}\right)$ is convergent in $D\left(\delta_{1}\right)$. Clearly, it must converge to $B$. Moreover $|B(1)|=1$ because evaluation at 1 is a continuous functional on $D\left(\delta_{1}\right)$.

Note that if the sequence $\left(z_{n}\right)$ is a Blaschke sequence bounded away from 1 , then (L) holds because then the factor $\left|1-z_{n}\right|^{2}$ is bounded away from zero.

The following proposition is a particular case of a theorem in 4] (page 205), based on a result from [1] (Corollary 3.5, page 858). The proof given here uses Carleson's interpolation theorem.

Proposition 2.3. Let $\left(z_{n}\right)$ be a sequence in $\mathbb{D}$. Then these two conditions are equivalent:

(1) any idempotent from $l^{\infty}$ can be interpolated over $\left(z_{n}\right)$ by a function from $H^{\infty}$,

(2) $\left(z_{n}\right)$ satisfies (C).

Proof. (2) implies (1) by Carleson's interpolation theorem. (11) implies (2): if (1) holds, then $\left(z_{n}\right)$ must be a Blaschke sequence. Assume now that (2n) does not hold. Then, for some subsequence $\left(z_{n_{k}}\right)$ we have that $\beta_{n_{k}}\left(z_{n_{k}}\right)$ converges to zero. Taking a further subsequence if necessary, we can assume that $\left(z_{n_{k}}\right)$ is a Carleson sequence. Let $\Gamma$ be the Blaschke product associated with $\left(z_{n_{k}}\right)$ and $\gamma_{k}$ be the product obtained by deleting the factor associated with $z_{n_{k}}$ from $\Gamma$. Assume that $f \in H^{\infty}$ interpolates the idempotent which is 1 on the terms of $\left(z_{n_{k}}\right)$ and zero elsewhere in $\left(z_{n}\right)$. Then $f$ must be of the form $\tilde{B} h$ where $\tilde{B}$ is the Blaschke product 
associated with the terms of $\left(z_{n}\right)$ not belonging to $\left(z_{n_{k}}\right)$ and $h$ is a function in $H^{\infty}$. Thus, $f\left(z_{n_{k}}\right)=\tilde{B}\left(z_{n_{k}}\right) h\left(z_{n_{k}}\right)$ and

$$
\tilde{B}\left(z_{n_{k}}\right)=\frac{\beta_{n_{k}}\left(z_{n_{k}}\right)}{\gamma_{k}\left(z_{n_{k}}\right)}
$$

converges to zero because $\gamma_{k}\left(z_{n_{k}}\right)$ is bounded below. Since $h$ is bounded, $f\left(z_{n_{k}}\right)$ converges to zero, contradicting the assumption that $f\left(z_{n_{k}}\right)=1$ for all $k$.

Theorem 2.4. If any idempotent from $l^{\infty}$ can be interpolated over $\left(z_{n}\right)$ by a function from $M\left(\delta_{1}\right)$, then $\left(z_{n}\right)$ satisfies (C) and ( $(\mathrm{L})$. On the other hand if $\left(z_{n}\right)$ satisfies both of these conditions, then it is an interpolating sequence for $M\left(\delta_{1}\right)$.

Proof. If we can interpolate any idempotent by an $M\left(\delta_{1}\right)$ function, then by Proposition $2.3\left(z_{n}\right)$ satisfies (C), since $M\left(\delta_{1}\right) \subset H^{\infty}$. Thus, it must also satisfy (N). Assume that $\left(P\left(z_{n}\right)\right) \notin l^{1}$. Then it can be split into two disjoint subsequences $u_{k}=P\left(z_{n_{k}}\right)$ and $v_{l}=P\left(z_{n_{l}}\right)$, neither of which is in $l^{1}$. Define $\left(a_{n}\right)$ to be 1 on $\left(z_{n_{k}}\right)$ and 0 on $\left(z_{n_{l}}\right)$. Assume that $c+(z-1) f \in M\left(\delta_{1}\right)$ interpolates $\left(a_{n}\right)$. Then the values of $\left(z_{n}-1\right) f\left(z_{n}\right)$ must have absolute value greater than $1 / 2$ on either one or the other subsequence (or both) depending on the value of $c$. One must have $\left(\left(1-\left|z_{n}\right|^{2}\right)\left|f\left(z_{n}\right)\right|^{2}\right) \in l^{1}$ because $\left(z_{n}\right)$ satisfies (ND). This implies that either $\left(u_{k}\right)$ or $\left(v_{l}\right)$ is summable, a contradiction.

Assume now that $\left(z_{n}\right)$ satisfies both conditions and let $\left(a_{n}\right) \in l^{\infty}$. Let

$$
B(z)=\prod_{n} \frac{\left|z_{n}\right|}{z_{n}} \frac{z_{n}-z}{1-\overline{z_{n} z}}
$$

be the Blaschke product along $\left(z_{n}\right)$ and let $h \in H^{\infty}$ satisfy

$$
h\left(z_{n}\right)=a_{n} .
$$

It follows from Proposition 2.2 that $B \in D\left(\delta_{1}\right)$ with $|B(1)|=1$. Then the function $\overline{B(1)}(B(1)-B) h$ belongs to $M\left(\delta_{1}\right)$ and interpolates $\left(a_{n}\right)$.

The conditions (C) and (L) are independent. In fact, no radial sequence $\left(z_{n}\right)$ converging to 1 satisfies (L), since in that case $P\left(z_{n}\right) \rightarrow \infty$. In particular, no growth rate on $\left|z_{n}\right|$ will force $\left(z_{n}\right)$ to be an interpolating sequence for $M\left(\delta_{1}\right)$, unlike the situation for $H^{\infty}$ (see, for example, 4]) and for the multipliers of the Dirichlet space $D(m)$ where $m$ is the Lebesgue measure (see [5]).

On the other hand, let $\left(z_{n}\right)$ be a sequence satisfying (L). For each $n$ let $w_{n}$ be such that

$$
\left|\frac{z_{n}-w_{n}}{1-\bar{w}_{n} z_{n}}\right|<\frac{1}{n}
$$

and

$$
\left|P\left(w_{n}\right)-P\left(z_{n}\right)\right|<2^{-n} .
$$

Then the interlacing of these two sequences (i.e., $z_{1}, w_{1}, z_{2}, w_{2}, \ldots$ ) still satisfies (L) and does not satisfy (C).

Finally, there exist sequences satisfying both of these conditions. One trivial example is any Carleson sequence bounded away from 1, as noted above.

For any $\epsilon>0$ the set $\{z \in \mathbb{D}: P(z)<\epsilon\}$ is formed by those points of $\mathbb{D}$ outside of the circle with center at $2 \epsilon /(\epsilon+1)$ and touching 1 . These sets are non-empty and have 1 in their boundary, so there exist sequences $\left(z_{n}\right)$ which satisfy $(\mathrm{L}$ ) having 1 as 
a sublimit. Any such sequence must satisfy $\left|z_{n}\right| \rightarrow 1$, so it must have a subsequence satisfying (C).

\section{WEAK INTERPOLATING SEQUENCES}

We begin with an estimate for norms of multipliers.

Lemma 3.1. Let $\phi \in M\left(\delta_{1}\right)$ be of the form $\phi(z)=(z-1) \psi(z)$ where $\psi \in H^{2}$ (i.e., $\phi(1)=0)$. Then

$$
\|\phi\|_{M} \leq 2\|\phi\|_{H^{\infty}}+N\|\psi\|_{H^{2}}
$$

where $N$ is the norm of the functional of evaluation at 1 on $D\left(\delta_{1}\right)$.

Proof. Let $f(z)=f(1)+(z-1) f_{1}(z)$ with $f_{1}$ in $H^{2}$. We have

$$
\|\phi f\|^{2}=\|\phi f\|_{H^{2}}^{2}+\|\psi f\|_{H^{2}}^{2}
$$

so

$$
\begin{aligned}
\|\phi f\| & \leq\|\phi f\|_{H^{2}}+\|\psi f\|_{H^{2}} \\
\|\phi f\|_{H^{2}} & \leq\|\phi\|_{H^{\infty}}\|f\|_{H^{2}} \\
\|\psi f\|_{H^{2}} & =\left\|\psi f(1)+\phi f_{1}\right\|_{H^{2}} \\
& \leq\|f(1) \psi\|_{H^{2}}+\|\phi\|_{H^{\infty}}\left\|f_{1}\right\|_{H^{2}}, \\
\|f(1) \psi\|_{H^{2}} & =\mid f(1)\|\| \psi \|_{H^{2}} \\
& \leq N\|f\|\|\psi\|_{H^{2}} .
\end{aligned}
$$

Thus,

$$
\|\phi f\| \leq\left(2\|\phi\|_{H^{\infty}}+N\|\psi\|_{H^{2}}\right)\|f\| .
$$

Theorem 3.2. A sequence $\left(z_{n}\right) \subset \mathbb{D}$ is a weak interpolating sequence for $M\left(\delta_{1}\right)$ if and only if it satisfies (C) and

$$
\left(P\left(z_{n}\right)\right) \in l^{\infty} .
$$

Proof. Sufficiency: Assume that (B) and (C) hold. Define

$$
\phi_{n}(z)=\frac{1-\left|z_{n}\right|^{2}}{z_{n}-1} \frac{z-1}{1-\overline{z_{n}} z} \frac{\beta_{n}(z)}{\beta_{n}\left(z_{n}\right)}
$$

and

$$
\psi_{n}(z)=\frac{\phi_{n}(z)}{z-1}
$$

The sequence $\left(\phi_{n}\right)$ is a sequence of multipliers of $D\left(\delta_{1}\right)$ and satisfies

$$
\phi_{n}\left(z_{m}\right)=\delta_{m n},
$$

and it remains to prove that it is bounded. By Lemma 3.1 it is enough to prove that $\left(\phi_{n}\right)$ and $\left(\psi_{n}\right)$ are bounded sequences in $H^{\infty}$ and $H^{2}$, respectively. We have

$$
\phi_{n}(z)=\frac{1-\left|z_{n}\right|^{2}}{z_{n}-1} \frac{z-z_{n}}{1-\overline{z_{n} z}} \frac{\beta_{n}(z)}{\beta_{n}\left(z_{n}\right)}+\frac{1-\left|z_{n}\right|^{2}}{z_{n}-1} \frac{z_{n}-1}{1-\overline{z_{n}} z} \frac{\beta_{n}(z)}{\beta_{n}\left(z_{n}\right)} .
$$

The first summand equals

$$
-\frac{z-z_{n}}{1-\overline{z_{n} z}} \frac{\beta_{n}(z)}{\beta_{n}\left(z_{n}\right)}\left(1-\overline{z_{n}}\right) P\left(z_{n}\right),
$$


whose absolute value is bounded by

$$
\frac{2 P\left(z_{n}\right)}{\left|\beta_{n}\left(z_{n}\right)\right|}
$$

and the second summand has absolute value bounded by

$$
\frac{2}{\left|\beta_{n}\left(z_{n}\right)\right|}
$$

since

$$
\left|\frac{1-\left|z_{n}\right|^{2}}{1-\overline{z_{n}} z}\right| \leq 1+\left|z_{n}\right|
$$

Moreover,

$$
\left\|\psi_{n}\right\|_{H^{2}}^{2}=\frac{\left(1-\left|z_{n}\right|^{2}\right)^{2}}{\left|1-z_{n}\right|^{2}\left|\beta_{n}\left(z_{n}\right)\right|^{2}}\left\|\frac{1}{1-\overline{z_{n} z}}\right\|_{H^{2}}^{2}=\frac{P\left(z_{n}\right)}{\left|\beta_{n}\left(z_{n}\right)\right|^{2}} .
$$

Necessity: By the initial observation that $\|\phi\|_{\infty} \leq\|\phi\|_{M}$ for multipliers, a bounded sequence in a multiplier algebra will be uniformly bounded as well. Therefore, in this particular case, a weak interpolating sequence for $M\left(\delta_{1}\right)$ must also be a weak interpolating sequence for $H^{\infty}$. By Carleson's theorem, such a sequence must satisfy (C).

Let

$$
\phi_{n}(z)=a_{n}+(z-1) f_{n}(z)
$$

be a bounded sequence of multipliers of $D\left(\delta_{1}\right)$ such that (3.2) holds.

Assume first that $\left|a_{n}-1\right|<1 / 2$. Then, by (3.2), for $m \neq n$

$$
a_{n}+\left(z_{m}-1\right) f_{n}\left(z_{m}\right)=0
$$

SO

$$
\frac{1}{2}<\left|\left(z_{m}-1\right) f_{n}\left(z_{m}\right)\right| \leq\left|z_{m}-1\right| \frac{|| f_{n} \|_{H^{2}}}{\left(1-\left|z_{m}\right|^{2}\right)^{\frac{1}{2}}}
$$

which gives

$$
P\left(z_{m}\right)=\frac{1-\left|z_{m}\right|^{2}}{\left|1-z_{m}\right|^{2}}<4|| f_{n} \|_{H^{2}}^{2},
$$

which is bounded independently of $n$.

So, if there is such a term in the sequence, then (B) holds. If not, then for all $n$, $\left|a_{n}-1\right| \geq 1 / 2$. But by similar calculations, only this time evaluating at $z_{n}$,

$$
P\left(z_{n}\right) \leq 4\left\|f_{n}\right\|_{H^{2}}^{2}
$$

Condition (B) is strictly weaker than (L), but analogous considerations to the ones closing the preceding section apply again. The difference is that in order to be a weak interpolating sequence, a Carleson sequence just has to remain outside of some circle $\{z \in \mathbb{D}: P(z)=r\}$ for some positive $r$. For example, any sequence $\left(z_{n}\right)$ converging to 1 and lying on such a circle will have subsequences which are weakly interpolating for $M\left(\delta_{1}\right)$. By Theorem [2.4, no such subsequences can be interpolating sequences $\left(P\left(z_{n}\right)\right.$ is constant). 


\section{Finitely Atomic Measures}

If

$$
\mu=\sum_{k=1}^{N} \mu_{k} \delta_{\lambda_{k}}
$$

is a finitely atomic measure on $\partial \mathbb{D}$, then $D(\mu)$ coincides as a set with the intersection of the sets $D\left(\delta_{\lambda_{k}}\right)$. Therefore, the multiplier algebra $M(\mu)$ contains the intersection $\bigcap_{k=1}^{N} M\left(\delta_{\lambda_{k}}\right)$ and must be equal to it because constants belong to $D(\mu)$, so an element from $M(\mu)$ is a bounded element from $D(\mu)$ and therefore belongs to each of the multiplier algebras of $D\left(\delta_{\lambda_{k}}\right)$.

Being contained in each of the algebras $M\left(\delta_{\lambda_{k}}\right), M(\mu)$ inherits a norm $\|\cdot\|_{k}$ from each of them. Given $\phi \in M(\mu)$, define the norm

$$
\|\phi\|_{s}=\sum_{k=1}^{N}\|\phi\|_{M\left(\delta_{\lambda_{k}}\right)} .
$$

It can easily be seen that this norm is equivalent to the operator norm on $M(\mu)$.

These observations allow us to generalize the two previous theorems to the case where $\mu$ is of the form (4.1).

Theorem 4.1. The (weak) interpolating sequences for $M(\mu)$ are the sequences $\left(z_{n}\right)$ which are (weak) interpolating sequences for $M\left(\delta_{\lambda_{k}}\right)$ for $1 \leq k \leq N$.

Proof. The equivalence of the norms $\|\cdot\|_{M(\mu)}$ and $\|\cdot\|_{s}$ and the fact that $M(\mu) \subset$ $M\left(\delta_{\lambda_{k}}\right)$ for each $k$ show that the second condition is necessary in both cases.

Let $\left(z_{n}\right)$ be an interpolating sequence for each $M\left(\delta_{\lambda_{k}}\right)$. In the proof of Theorem 2.4 we saw that for each bounded sequence $\left(a_{n}\right)$ there exists an interpolator of the form $\left(z-\lambda_{k}\right) h(z)$ where $h \in H^{2}$. Also, any bounded function of this form belongs to $M\left(\delta_{\lambda_{k}}\right)$. Thus, given $\left(a_{n}\right)$, let $\phi_{1}(z)=\left(z-\lambda_{1}\right) h_{1}(z)$ be an interpolator of $\left(a_{n}\right)$ in $M\left(\delta_{\lambda_{1}}\right)$ and for $k \geq 2$ let $\phi_{k}(z)=\left(z-\lambda_{k}\right) h_{k}(z)$ be interpolators of the constant sequence 1 in $M\left(\delta_{\lambda_{k}}\right)$. The product of all these functions is an interpolator of $\left(a_{n}\right)$ in $M(\mu)$.

Now let $\left(z_{n}\right)$ be a weak interpolating sequence for each $M\left(\delta_{\lambda_{k}}\right)$. For each $k$, let $\left(\phi_{k, n}\right)$ be a bounded sequence in $M\left(\delta_{\lambda_{k}}\right)$ such that $\phi_{k, n}\left(z_{m}\right)=\delta_{m n}$. We saw in the proof of Theorem 3.2 that we can choose $\phi_{k, n}$ to be of the form $\left(z-\lambda_{k}\right) h_{k, n}(z)$ with $h_{k, n} \in H^{2}$. Let $\phi_{n}(z)=\prod_{k=1}^{N} \phi_{k, n}(z)$. This defines a sequence in $M(\mu)$ such that $\phi_{n}\left(z_{m}\right)=\delta_{m n}$, and it only remains to see that it is bounded. It is enough to see that it is bounded in each $M\left(\delta_{\lambda_{k}}\right)$. We will use Lemma 3.1. For each $k$ the sequence $\left(\phi_{k, n}\right)$ is bounded in $H^{\infty}$ because the $H^{\infty}$ norm is dominated by the multiplier norm. Therefore $\left(\phi_{n}\right)$ is bounded in $H^{\infty}$. It only remains to prove that for each $k$ the sequence $\left(\phi_{n} /\left(z-\lambda_{k}\right)\right)$ is bounded in $H^{2}$. But for each $k$,

$$
\frac{\phi_{n}(z)}{z-\lambda_{k}}=\left(\prod_{j \neq k} \phi_{j, n}(z)\right) h_{k, n}(z)
$$

and the first factor on the right is a bounded sequence in $H^{\infty}$, and $\left(h_{k, n}\right)$ is bounded in $H^{2}$. 


\section{ACKNOWLEDGEMENTS}

The author thanks his advisor, Donald Sarason, for the suggestion of this topic and many helpful conversations, as well as several careful readings of the manuscript, with suggestions for improvement. This paper was also inspired by a talk given by Alexander Schuster in the Analysis Seminar of the Department of Mathematics at the University of California, Berkeley, in the Fall of 1999.

\section{REFERENCES}

[1] William G. Bade and Philip C. Curtis, Jr. The Wedderburn decomposition of commutative Banach algebras. Amer. J. Math., 82:851-866, 1960. MR 23:A529

[2] Lennart Carleson. An interpolation problem for bounded analytic functions. Amer. J. Math., 80:921-930, 1958. MR 22:8129

[3] John B. Garnett. Bounded analytic functions. Academic Press, Inc. [Harcourt Brace Jovanovich Publishers], New York, 1981. MR 83g:30037

[4] Kenneth Hoffman. Banach spaces of analytic functions. Dover Publications Inc., New York, 1988. Reprint of the 1962 original. MR 92d:46066

[5] Donald Marshall and Carl Sundberg. Interpolating sequences for the multipliers of the Dirichlet space. Preprint.

[6] Stefan Richter. A representation theorem for cyclic analytic two-isometries. Trans. Amer. Math. Soc., 328(1):325-349, 1991. MR 92e:47052

[7] Stefan Richter and Carl Sundberg. A formula for the local Dirichlet integral. Michigan Math. J., 38(3):355-379, 1991. MR 92i:47035

[8] Donald Sarason. Doubly shift-invariant spaces in $H^{2}$. J. Operator Theory, 16(1):75-97, 1986. MR 88d:47014b

[9] Donald Sarason. Local Dirichlet spaces as de Branges-Rovnyak spaces. Proc. Amer. Math. Soc., 125(7):2133-2139, 1997. MR 98h:46023

[10] H. S. Shapiro and A. L. Shields. On some interpolation problems for analytic functions. Amer. J. Math., 83:513-532, 1961. MR 24:A3280

Department of Mathematics, University of California, Berkeley, California 94720 Current address: Departamento de Matemática-IST, Av. Rovisco Pais, 1049-001 Lisboa, Portugal

E-mail address: aserra@math.ist.utl.pt 